\title{
Corrugation Enabled Asymmetrically Ultrastretchable (95\%) Monocrystalline Silicon Solar Cells with High Efficiency (19\%)
}

\author{
Nazek El-Atab, Nadeem Qaiser, Rabab Bahabry, and Muhammad Mustafa Hussain*
}

Stretchable solar cells are of growing interest due their key role in realizing many applications such as wearables and biomedical devices. Ultrastretchability, high energy-efficiency, biocompatibility, and mechanical resilience are essential characteristics of such energy harvesting devices. Here, the development of wafer-scale monocrystalline silicon solar cells with world-record ultrastretchability $(95 \%)$ and efficiency $(19 \%)$ is demonstrated using a laser-patterning based corrugation technique. The demonstrated approach transforms interdigitated back contacts (IBC) based rigid solar cells into mechanically reliable but ultrastretchable cells with negligible degradation in the electric performance in terms of current density, opencircuit voltage, and fill factor. The corrugation method is based on the creation of alternating grooves resulting in silicon islands with different shapes. The stretchability is achieved by orthogonally aligning the active silicon islands to the applied tensile stress and using a biocompatible elastomer (Ecoflex) as a stretchable substrate. The resulting mechanics ensure that the brittle silicon areas do not experience significant mechanical stresses upon asymmetrical stretching. Different patterns are studied including linear, diamond, and triangular patterns, each of which results in a different stretchability and loss of active silicon area. Finally, finite element method based simulation is conducted to study the generated deformation in the different patterned solar cells.

skin, ${ }^{[3,4]}$ stretchable displays, ${ }^{[5,6]}$ and so on. In particular, mechanically deformable energy-harvesting ${ }^{[7,8]}$ and energystorage $^{[9-11]}$ devices are crucial for achieving efficacy and portability. Unlike flexibility which can be achieved by thinning down the material such that the generated strain is below its fracture limit, stretchability may involve out-of-plane deformation and reversible change of material size and thus should be tackled differently. ${ }^{[12]}$ The early demonstrations of stretchable energy devices such as batteries $^{[13]}$ and supercapacitors ${ }^{[14]}$ are based on the dispersion of electronic components in inherently elastic materials such as elastomers. Bao and co-workers reported the first intrinsically stretchable solar cell using organic materials which can accommodate reversible strains up to $27 \%$ with a power conversion efficiency of $\approx 2 \%$. ${ }^{[15]}$ The development of different organic-based elastic photovoltaics followed; however, the major drawbacks lie in their environmental instability and low efficacy (below 8\%). ${ }^{[16-18]}$

In contrast, semiconductor based solar cells show higher efficiencies; however,

The rapid and substantial progress in the development of different stretchable electronics has recently stimulated strong research interest. Examples include wearables, ${ }^{[1,2]}$ artificial

Dr. N. El-Atab, Dr. N. Qaiser, Prof. M. M. Hussain

$\mathrm{MMH}$ Labs

Electrical Engineering

Computer Electrical Mathematical Science and Engineering Division

King Abdullah University of Science and Technology (KAUST)

Thuwal 23955-6900, Saudi Arabia

E-mail: muhammad.hussain@kaust.edu.sa; mmhussain@berkeley.edu

Dr. R. Bahabry

Department of Physics

University of Jeddah

Jeddah 21589-80200, Saudi Arabia

Prof. M. M. Hussain

EECS

University of California

Berkeley 94720, CA, USA

The ORCID identification number(s) for the author(s) of this article can be found under https://doi.org/10.1002/aenm.201902883.

DOI: 10.1002/aenm.201902883 they are inherently rigid and brittle. To overcome these constraints, various forms of shape engineering were developed such as serpentine, wavy, and stiff-island structures ${ }^{[19-21]}$ which can achieve stretching due to different mechanisms such as out-of-plane deformation, buckling, and twisting. ${ }^{[22]}$ The first inorganic semiconductor-based stretchable solar cell was reported by Rogers and co-workers where single junction GaAs microcells (3.6- $\mu \mathrm{m}$-thick) were transferred onto a prestrained elastomer with downward buckled interconnects resulting in an efficiency of $\approx 13 \%$ with strains up to $20 \%$. ${ }^{[23]}$ In a follow-up work, the authors used ultrathin and geometrically structured dual junction GaInP-GaAs microcells to achieve 60\% stretchability and $19 \%$ efficiency at the expense of a $33 \%$ loss of active area. ${ }^{[24]}$ However, all of the demonstrated inorganic semiconductor based stretchable photovoltaics necessitate an aligned transfer printing of the ultrathin and patterned inorganic material from a crystalline wafer onto a prestrained elastic substrate with good adhesion and low alignment mismatch. ${ }^{\text {[25] }}$ Previously, we demonstrated a deep reactive ion etching (DRIE) based linear-corrugation technique to convert rigid solar cells 

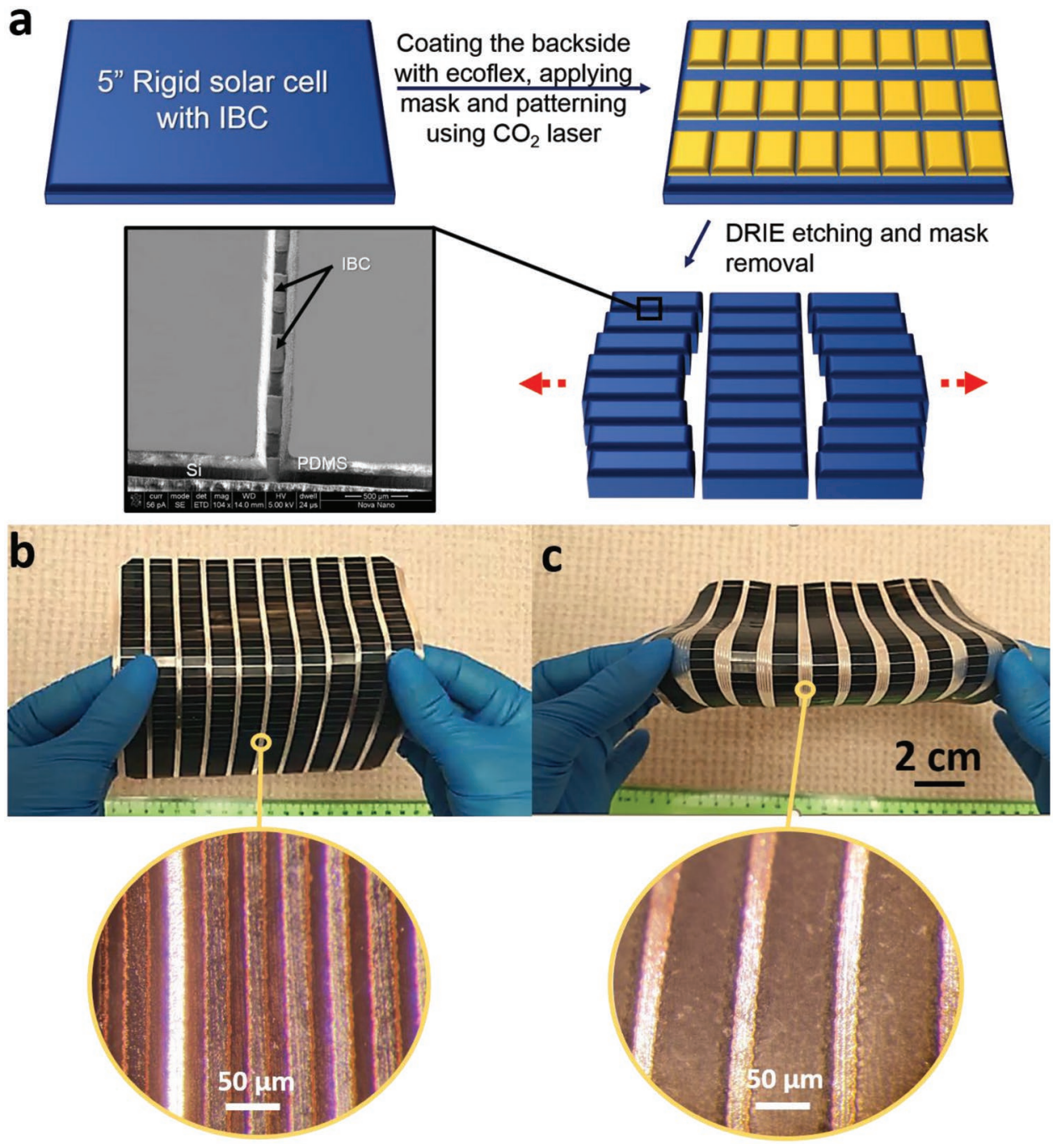

Figure 1. a) Fabrication process flow of the stretchable solar cells. Inset shows SEM cross section of the solar cell and the IC structures connecting two silicon islands. Optical image of the stretchable solar cell b) before stretching and c) after stretching by $\approx 33 \%$. Insets show that IBC structures on Ecoflex in between the islands before and after stretching, respectively.

with interdigitated back contacts (IBC) into one-directional flexible cells (perpendicularly to the IBC grid) with no degradation in the efficiency. ${ }^{[26]}$ Here, we demonstrate the transformation of commercial, wafer-scale and IBC-based rigid silicon solar cells into ultrastretchable ones by simply coating the cells with a biocompatible elastomer followed by applying a corrugation technique. ${ }^{[26]}$ Different corrugation patterns including linear, diamond, and triangular patterns are studied to minimize the strain experienced by the brittle silicon areas upon asymmetrical stretching, parallel to the IBC grid. The demonstrated approach thus enables the stretchability of the rigid and thick solar cells $(\approx 170-\mu \mathrm{m}$-thick) with negligible degradation in the original electrical performance. Finally, it is worth to note that the highest reported silicon-based photovoltaics efficiencies $(>25 \%)$ used the IBC architecture, ${ }^{[27-32]}$ thus the proposed approach contributes towards the development of ultrastretchable silicon solar cells with the highest efficiencies.

The stretching mechanism is demonstrated on wafer scale (25 in. ${ }^{2}$ ), commercial and rigid monocrystalline silicon solar cells with IBC. The front side of the rigid solar cells was initially coated with a $160-\mu \mathrm{m}$-thick polydimethylsiloxane (PDMS) polymeric material as a hard mask followed by its patterning using $\mathrm{CO}_{2}$ laser while the back side is coated with an elastic biocompatible elastomer (Ecoflex, 200- $\mu$ m-thick). The sample is then inserted into a DRIE system where the exposed areas are completely etched until the IBC is reached (Figure 1a). The silicon microcells are aligned along the IBC orthogonally to the applied tensile stress to enable the stretching of the structure while minimizing the strain in the silicon areas. The grooves between the microcells are $\approx 140 \mu \mathrm{m}$ wide (Figure $1 \mathrm{a}$, inset) while the spacing between two consecutive lines of silicon cells is $1 \mathrm{~mm}$. Figure 1b,c shows the prestretched and stretched wafer-scale solar cell when an asymmetrical tensile stress is applied near its center orthogonally to the IBC. As a result, the elastomer absorbs most of the applied strain while the silicon and IBC experience negligible strains, as shown in the Figure $1 \mathrm{~b}$ (insets), where the elastomer is stretched by more than $\approx 120 \%$ while the complete structure with linear grooves is stretched 
by $\approx 33 \%$. The demonstrated approach enable out-of-plane stretching which is required for different applications such as stretching and wrapping around curved objects or robotic joints (Videos S1-S3, Supporting Information). However, it should be noted that due to the linear structure of the IBC, only uniaxial stretching perpendicularly to the IBC is possible in this case. However, the demonstration in this work shows that designing the IBC with different fractal designs could enable a multiaxial stretching capability. Different patterns are also studied to allow for a higher asymmetrical stretchability including diamond and triangular patterns with different spacing. In fact, when an asymmetrical tensile stress is applied across a specific area, the surrounding silicon islands experience a compressive strain which, above a critical value, result in diagonal cracks (Figure S1, Supporting Information). Thus, patterned islands assist in minimizing and relieving the compressive strain experienced by the surrounding islands when larger asymmetrical tensile stresses are applied across a specific region.

The electrical performance of the different patterned stretchable solar cells in terms of current density-voltage $(J-V)$ and power density-voltage $(P-V)$ characteristics are studied under 1 sun under a solar simulator in air (spectral mismatch correction is conducted at room temperature, standard test conditions). The measured $J-V$ and $P-V$ characteristics (Figure 2a,b), normalized to the active silicon area, confirm that the applied corrugation technique enables the transformation of rigid solar cells into stretchable ones with negligible degradation in the performance where the measured $J_{\mathrm{sc}}=38.75 \mathrm{~mA} \mathrm{~cm}^{-2}$, $V_{\text {oc }}=0.63, \mathrm{FF}=75 \%$ and efficiency $\eta=18.8 \%$. The DRIE recipe is optimized (low flow rate of $\mathrm{SF}_{6}$, high flow rate of $\mathrm{C}_{4} \mathrm{~F}_{8}$ and low pressure, ${ }^{[33]}$ see the Experimental Section) to minimize sidewall damage during the process as confirmed by the observed negligible overall degradation in efficiency. Moreover, three effects in the demonstrated corrugated cells can counteract the DRIE damage effect. First, the $\mathrm{C}_{4} \mathrm{~F}_{8}$ gas during DRIE results in the deposition of a fluorocarbon-based polymer on the sidewalls, which contributes in passivating the silicon sidewalls and thus reduces potential surface recombination. ${ }^{[34]}$ Moreover, the exposure of sidewalls/junction increases photons absorption and light-generated current. ${ }^{[35]}$ Finally, the corrugated solar cell with fin-like architecture enhances the thermal dissipation in solar cells due to the increased surface area-to-volume ratio. ${ }^{[36]}$ The Ecoflex transparency is also studied and shown to be dependent on its thickness where the transmission characteristic of around $200-\mu \mathrm{m}$-thick elastomer is comparable to that of PDMS (Figure 2c). This characteristic becomes critical for applications of semitransparent solar cells such as greenhouse roof application to enable sunlight to enter the greenhouse for plant photosynthesis. ${ }^{[37]}$

The stretchability of the different studied patterns shows that using the same design but with larger spacing, a higher asymmetrical stretching can be achieved due to the larger elastomer area which can absorb higher strains. For instance, the diamond patterned solar cells with 1 and $2 \mathrm{~mm}$ spacing show $51 \%$ and $75 \%$ stretchability, respectively (Figure $2 \mathrm{~d}$ ); however, this comes at the expense of a higher loss of active silicon area (12\% for $1 \mathrm{~mm}$ vs $17 \%$ for $2 \mathrm{~mm}$ spacing). Nevertheless, using different patterns that allow the relief and minimization of the generated strain during the applied tensile force enable higher asymmetrical stretching for the same or lower loss of active silicon area. For instance, when comparing the triangular patterned solar cell with $1 \mathrm{~mm}$ spacing and the diamond patterned solar cell with $2 \mathrm{~mm}$ spacing, it can be seen that the former option results in a $95 \%$ stretchability with $13.5 \%$ loss of silicon area while the latter design allow a $75 \%$ stretchability with $17 \%$ loss of active silicon area (Figure 2d). It is worth to mention that the triangular pattern combines the linear pattern with the diamond one. The performance of the triangular patterned stretchable solar cells and the resistance across a single IBC are also studied when stretched up to $65 \%$ and up to 500 cycles (with $30 \%$ stretching). The results confirm that the corrugation technique results in mechanically robust stretchable cells (Figure 2e,f), which is an essential characteristic for applications such as wearables and biomedical devices.

The different patterned solar cells before and after applying an asymmetrical tensile stress are demonstrated in Figure 3. The strain is mostly absorbed by the elastomer where it is stretched by $\approx 1050 \%$ to enable a $95 \%$ stretchability of the complete solar cell with triangular corrugation (Figure 3). Even when the asymmetrical stretching is applied closer to the positive or negative terminals of the IBC grid, a high stretchability can still be achieved (Figure S2, Supporting Information) in addition to possible out-of-plane deformations (Figure S3, Supporting Information). As a result, the presented stretchable solar cells are promising for robotic joints application (Video S3, Supporting Information). The stretchability, efficiency, and area of the previously reported stretchable solar cells are summarized in Figure 4. It is worth to note that using GaInP-GaAs dual junction solar cells, the achieved efficiency was $19 \%$, however, at the expense of a $33 \%$ loss of active area (effective power conversion efficiency is $12.5 \%$ when total area is taken into consideration instead of active area). In the demonstrated stretchable silicon solar cells, 19\% efficiency was obtained with a $13 \%$ loss of active area (effective power conversion efficiency of $16.5 \%$ ), as a result, the actual power output for the same area is higher with the stretchable monocrystalline silicon cells.

Finally, to confirm the effect of the different patterns and spacing on the stretchability of the solar cells, the deformation contours in the different corrugated solar cells (linear, diamond, and triangular with $1 \mathrm{~mm}$ spacing) are studied using finite element methods (FEM) based simulations using COMSOL where the same stretching of $0.6 \mathrm{~mm}$ is imposed at the edges of the solar cells with the same area. The results show that the triangular patterned cells enable higher deformations and thus higher motion of the islands compared to the diamond cells (Figure 5). In contrast, for the diamond corrugated cells case, the free motion is constrained by the surrounding rigid islands which limit its stretchability. Moreover, the linear corrugated cells show higher strain due to the larger size and shape of the rigid rectangular islands (Figure S4, Supporting Information). It is also worth to note that increasing the spacing between the silicon islands reduces the strain experienced by the cells (Figure S5, Supporting Information), as a result, a higher stretchability can be achieved.

In conclusion, we have demonstrated a corrugation approach to transform wafer scale rigid solar cells with IBC into ultrastretchable ones with negligible degradation in the electrical performance. A world record stretchability (95\%) and efficiency 

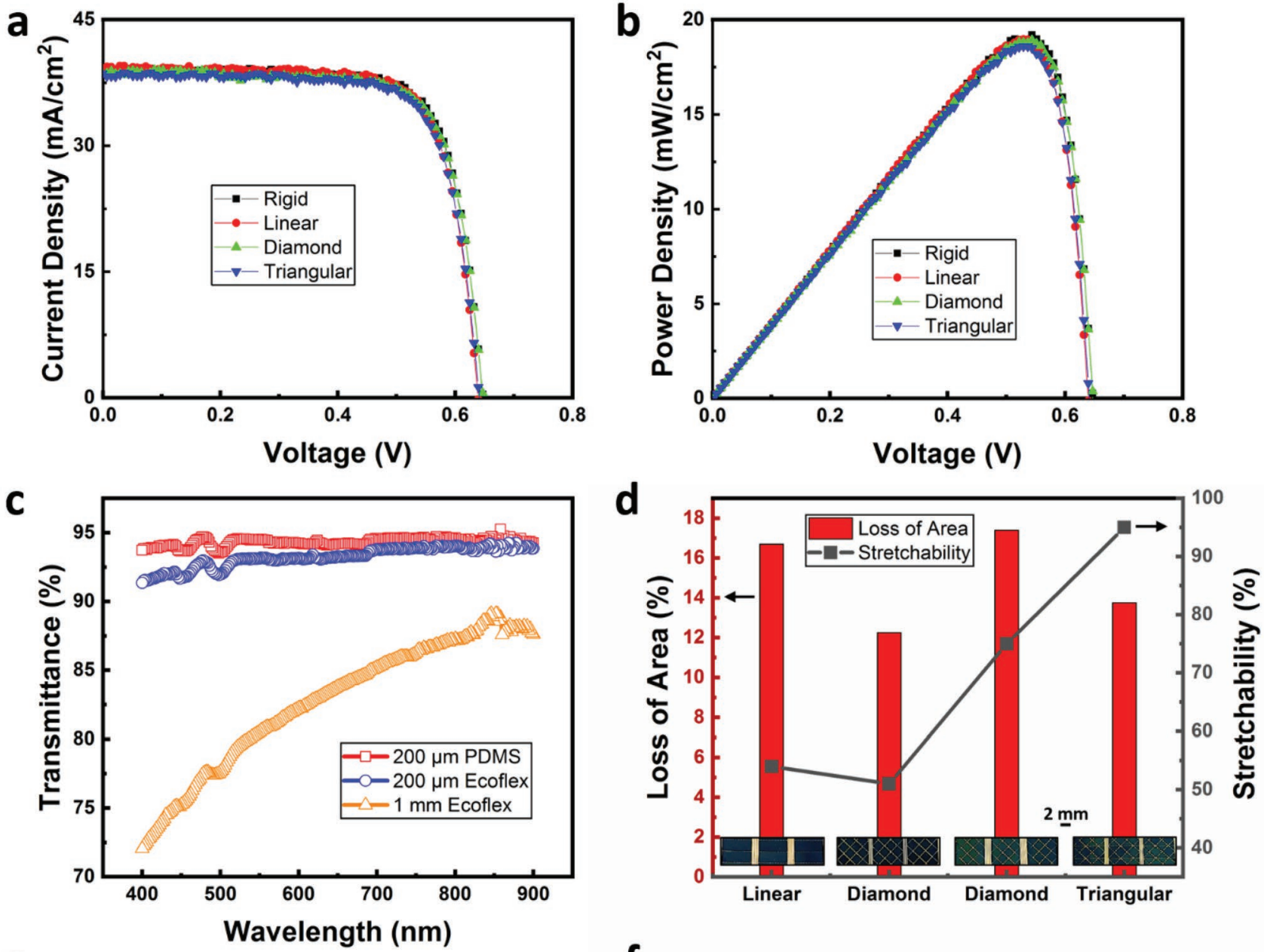

e
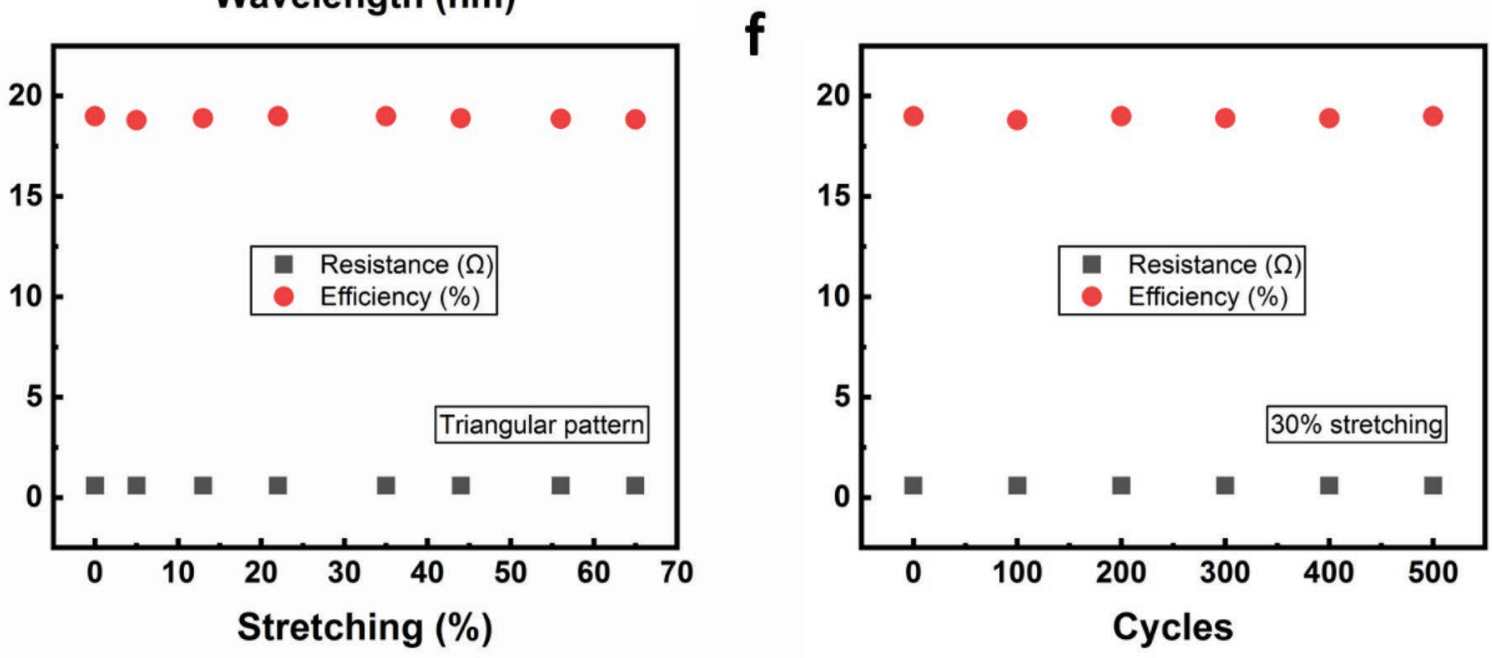

Figure 2. a) $J-V$ characteristic of the different patterned solar cells $(2.1 \mathrm{~cm}$ by $12.5 \mathrm{~cm})$ under 1 sun. Rigid cell represent the solar cell before applying the stretching technique, a negligible degradation of $\Delta \eta \approx 0.3 \%$ is observed. b) $P-V$ characteristic of the solar cells measured under 1 -sun. The results are normalized to the area of active silicon, taking into consideration the loss in the grooves. c) Transmittance characteristic of PDMS and Ecoflex with different thicknesses. d) Stretchability of the different patterned solar cells with different spacing and their corresponding loss of active silicon area. e) Measured efficiency and resistance of the IBC when stretched up to $65 \%$. f) Cycling test showing the efficiency and resistance of the IBC when stretched by $30 \%$ up to 500 cycles.

(19\%) are obtained. The stretching technique does not require aligned transfer printing of a patterned inorganic material onto an elastomer as reported by previous works, instead, the elastomer is simply coated on the backside of the rigid solar cell followed by patterning the silicon active area into islands which are readily interconnected using the IBC. The results show that, 


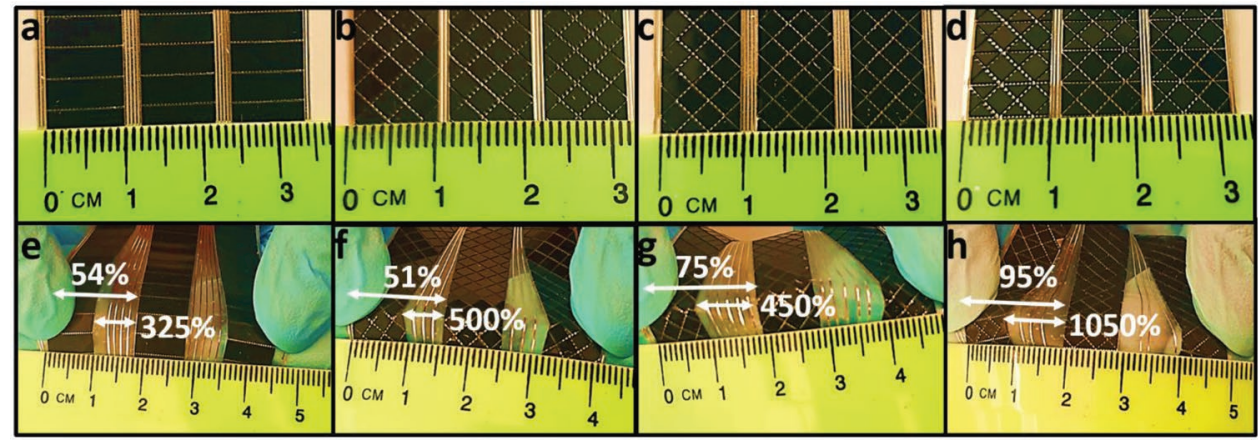

Figure 3. a) Linear patterned stretchable solar cells with $2 \mathrm{~mm}$ spacing. b) Diamond patterned solar cells with $1 \mathrm{~mm}$ spacing, c) Diamond patterned solar cells with $2 \mathrm{~mm}$ spacing, d) Triangular patterned solar cells with $1 \mathrm{~mm}$ spacing. e) Stretching of solar cell shown in Figure 1a by 54\% while the elastomer is stretched by $325 \%$, f) Stretching of solar cell shown in Figure 1 b by $51 \%$ while the elastomer is stretched by $500 \%$, g) Stretching of solar cell shown in Figure 1c by $75 \%$ while the elastomer is stretched by $450 \%$, h) Stretching of solar cell shown in Figure 1d by $95 \%$ while the elastomer is stretched by $1050 \%$.

by orthogonally aligning the rigid areas to the applied stress, the strain is mostly absorbed by the elastomer while the brittle silicon areas experience negligible strain. The demonstration also shows that different shapes of islands allow the relief of the generated strain during the application of the tensile force, as a result, higher stretchabilities can be achieved with lower losses of active silicon area. The patterned solar cells are simulated using COMSOL which confirmed the larger deformation with the triangular patterns compared to the diamond and linear patterns where the free motion of the islands is constrained by the surrounding silicon areas. Stretchable solar cells with higher efficiencies (>25\%) can be obtained by applying the demonstrated approach on the recently demonstrated IBC based solar cells with improved electrical performance. Finally, multiaxial stretching capability can be achieved in solar cells using IBC structures with different fractal designs.

\section{Experimental Section}

Fabrication of the Ultrastretchable Solar Cells: The stretchable solar cells were fabricated using a corrugation method consisting of laser patterning followed by DRIE etching. The stretching mechanism was applied on commercial wafer-scale ( 5 in. by 5 in.) IBC-based monocrystalline silicon solar cells and with high efficiency (19\%). First, the PDMS encapsulant was spin coated on the front side of the solar cell and cured at a temperature of cured at $60{ }^{\circ} \mathrm{C}$ for a duration of $2 \mathrm{~h}$ to obtain $160 \mu \mathrm{m}$ of PDMS hard mask. The PDMS was arranged by mixing 10:1 base silicone elastomer (Sylgard 184): curing agent. A vacuum mixer was used (THINKY Planetary Vacuum Mixer) to mix the PDMS for 4 min with a rotation speed of $700 \mathrm{rpm}$ at a pressure of $1.1 \mathrm{kPa}$ to remove any entrapped air. To pattern the PDMS hard mask, a $\mathrm{CO}_{2}$ laser (Universal Laser Systems PLS6.75) was used, the speed of the laser was set to $40 \mathrm{~mm} \mathrm{~s}^{-2}$, while the power was set to $24 \mathrm{~W}$ and the used z-height is $1 \mathrm{~mm}$. The backside of the solar cell was then coated with $200 \mu \mathrm{m}$ Ecoflex 00-30 using the spin coating technique. The Ecoflex was prepared by manually mixing 1A:1B parts in a 1:1 weight ratio. The solar cell was then heated up to $60{ }^{\circ} \mathrm{C}$ for $2 \mathrm{~h}$ for the Ecoflex to cure. The exposed area on the front side of the cell was then etched in a DRIE at $-20{ }^{\circ} \mathrm{C}$ using sulfur hexafluoride $\left(\mathrm{SF}_{6}\right)$ with a flow rate of 5 and $100 \mathrm{sccm}$ for the deposition and etching steps, respectively, and carbon fluoride $\left(\mathrm{C}_{4} \mathrm{~F}_{8}\right)$ with a flow rate of 100 and $5 \mathrm{sccm}$ for the deposition and etching steps, respectively, at a pressure of 30 mTorr. The percentage of active silicon area lost due to the corrugation technique was calculated using an image processing software (KLONK). Scanning electron microscopy images were acquired using an SEM NOVA system with an acceleration voltage of $5 \mathrm{kV}$ and a current of $56 \mathrm{pA}$.

Characterizations and Measurements: The $J-V$ and $P-V$ curves were measured and calculated using a solar simulator in air (Newport,

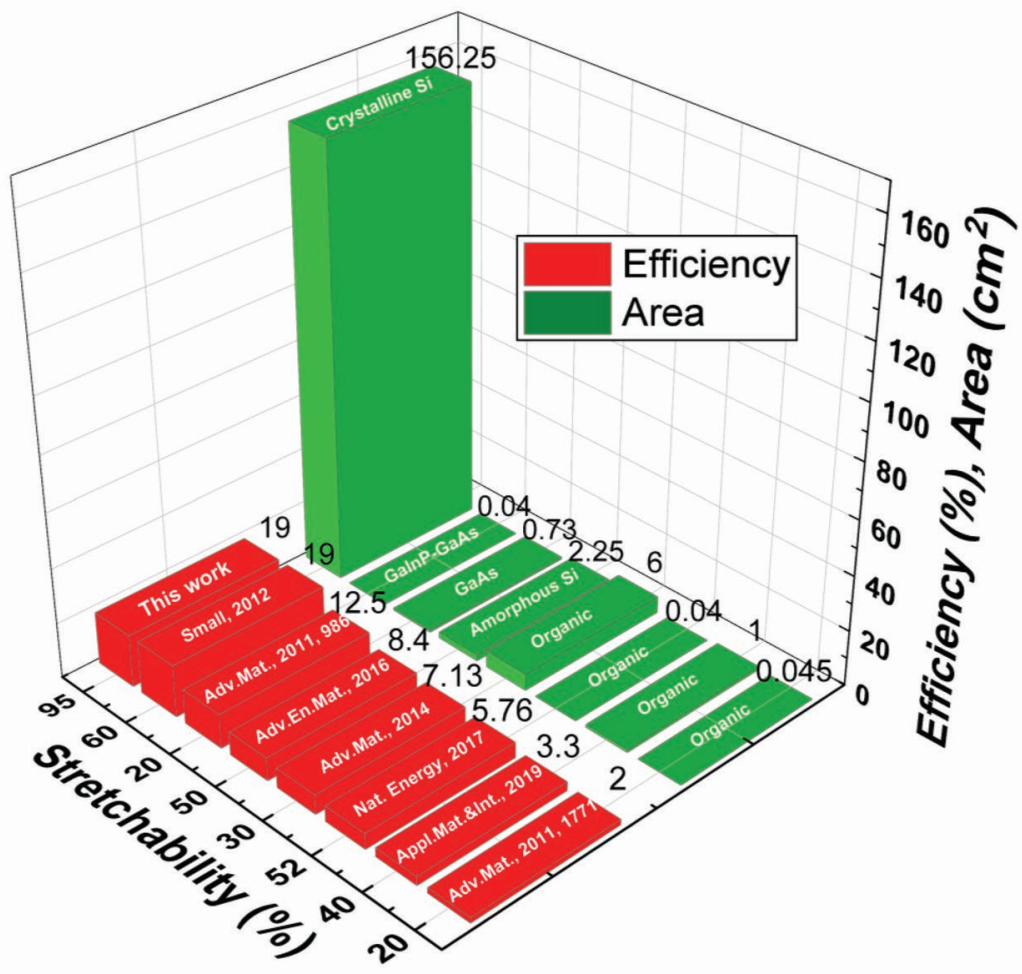

Figure 4. Comparison between previously reported stretchable solar cells ${ }^{[15-18,23-25]}$ and this work in terms of stretchability, efficiency, and area. The solar cells using inorganic semiconductor materials are mentioned. 
a

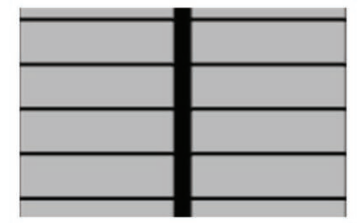

d

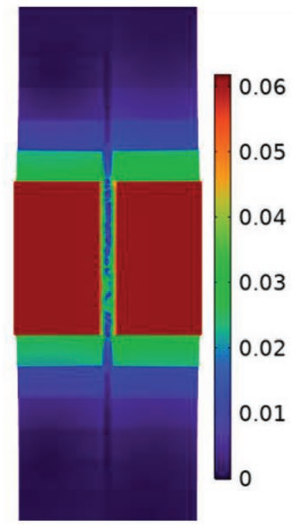

b

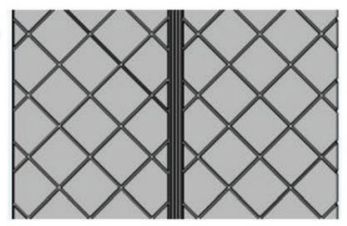

e

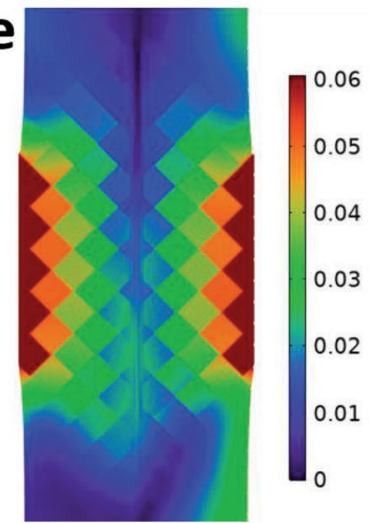

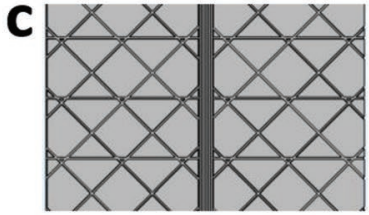

f

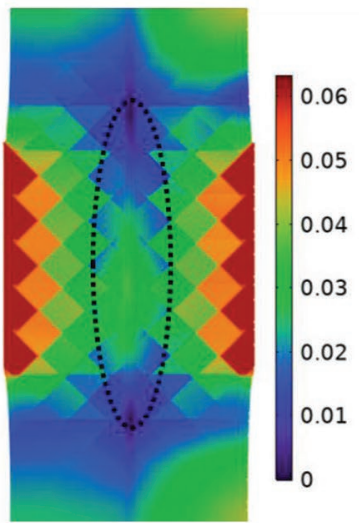

Figure 5. a) Linear, b) diamond, and c) triangular patterned solar cells with $1 \mathrm{~mm}$ spacing simulated using COMSOL. d) Simulated deformation contours in the structure shown in inset (a), e) Simulated deformation contours in the structure shown in inset (b), and f) Simulated deformation contours in the structure shown in inset (c), circled area shows increased deformation and therefore larger motion of islands in the triangular corrugated solar cell. A $0.6 \mathrm{~mm}$ displacement is applied for all three structures.

Oriel Class A, Sol3A) connected to a source meter (Keithley 2420-C) under 1 sun illumination with intensity of $1000 \mathrm{~W} \mathrm{~m}^{-2}$ (AM $1.5 \mathrm{G}$ ). The calibration was accomplished using a silicon-based reference cell (Newport certified). The $J-V$ curves were measured and calculated along the reverse scan direction: 1 to $-1.2 \mathrm{~V}$, with a step voltage of $7.35 \mathrm{mV}$ and scan speed of $82 \mathrm{mV} \mathrm{s}^{-1}$. The initial temperature of the characterized solar cells was preconditioned at $21{ }^{\circ} \mathrm{C}$ before exposing them to 1 sun under the solar simulator. Transmittance data on the PDMS and Ecoflex were measured using a Thermo Evolution 600 UV-Vis system.

COMSOL Simulations: 3D FEM analysis was used to replicate the mechanical response of the studied designs. The geometries were first designed in a CAD tool called SOLIDWORKS. All of the dimensions, i.e., thickness of the Si solar cell, Ecoflex substrate, and metal contacts were comparable to the fabricated samples. Three patterns are studied including linear, diamond and triangular corrugations with two different spacing: 1 and $2 \mathrm{~mm}$. The 3D designs were then imported to the COMSOL software for mechanical analysis. Mechanical deformation and induced principal strain as a function of imposed displacement or stretching were mapped using the "Solid Mechanics" module. To compare the experimental stretching of the samples, the displacement was prescribed at the edges of the sample. It is worthy to mention that to impose the displacement, the selected region or area taken was same for all of the designs. To accommodate the large deformation, "Geometric nonlinearity" was used. In order to ensure the solution convergence, a refined mesh was used. Elastic modulus and Poisson ratio of $\mathrm{Si}, \mathrm{Cu}$, and Ecoflex were taken as to be $170 \mathrm{GPa}, 120 \mathrm{GPa}$, and $750 \mathrm{kPa}, 0.28,0.34$, and 0.49 , respectively.

\section{Supporting Information}

Supporting Information is available from the Wiley Online Library or from the author.

\section{Acknowledgements}

M.M.H. conceived the idea and directed the project. N.E.-A. designed, fabricated, and characterized the stretchable solar cells and analyzed the results. N.Q. assisted in COMSOL simulations. All authors discussed the results. This publication is based upon work supported by the King Abdullah University of Science and Technology (KAUST) Office of Sponsored Research (OSR) under Award No. Sensor Innovation Initiative OSR-2015-Sensors-2707 and KAUST-KFUPM Special Initiative OSR-2016-KKI-2880.

\section{Conflict of Interest}

The authors declare no conflict of interest.

\section{Keywords}

corrugation, interdigitated back contacts, monocrystalline silicon, photovoltaics, stretchable electronics

Received: September 4, 2019

Revised: September 25, 2019

Published online:

[1] R. Matsuzaki, K. Tabayashi, Adv. Funct. Mater. 2015, 25, 3797.

[2] M. Mohammed, R. Kramer, Adv. Mater. 2017, 29, 1604965.

[3] J. Ge, L. Sun, F. Zhang, Y. Zhang, L. Shi, H. Zhao, H. Zhu, H. Jiang, S. Yu, Adv. Mater. 2016, 28, 783.

[4] Q. Hua, J. Sun, H. Liu, R. Bao, R. Yu, J. Zhai, C. Pan, Z. Wang, Nat. Commun. 2018, 9, 1.

[5] J. Koo, D. Kim, H. Shim, T. Kim, D. Kim, Adv. Funct. Mater. 2018, 28, 1801834

[6] N. Qaiser, S. Khan, K. Chow, M. Cordero, I. Wicaksono, M. Hussain, Adv. Mater. Technol. 2018, 3, 1800344.

[7] C. Jeong, J. Lee, S. Han, J. Ryu, G. Hwang, D. Park, J. Park, S. Lee, M. Byun, S. Ko, K. Lee, Adv. Mater. 2015, 27, 2866.

[8] D. Lipomi, Z. Bao, Energy Environ. Sci. 2011, 4, 3314. 
[9] N. Zhang, W. Zhou, Q. Zhang, P. Luan, L. Cai, F. Yang, X. Zhang, Q. Fan, W. Zhou, Z. Xiao, X. Gu, H. Chen, K. Li, S. Xiao, Y. Wang, H. Liu, S. Xie, Nanoscale 2015, 7, 12492.

[10] Y. Huang, M. Zhong, Y. Huang, M. Zhu, Z. Pei, Z. Wang, Q. Xue, X. Xie, C. Zhi, Nat. Commun. 2015, 6, 1.

[11] S. Pang, Y. Gao, S. Choi, Adv. Energy Mater. 2018, 8, 1702261.

[12] J. Rogers, T. Someya, Y. Huang, Science 2010, 327, 1603.

[13] M. Kaltenbrunner, G. Kettlgruber, C. Siket, R. Schwödiauer, S. Bauer, Adv. Mater. 2010, 22, 18.

[14] C. Yu, C. Masarapu, J. Rong, B. Wei, H. Jiang, Adv. Mater. 2009, 21, 4793.

[15] D. Lipomi, B. Tee, M. Vosgueritchian, Z. Bao, Adv. Mater. 2011, 23, 1771.

[16] H. Jinno, K. Fukuda, X. Xu, S. Park, Y. Suzuki, M. Koizumi, T. Yokota, I. Osaka, K. Takimiya, T. Someya, Nat. Energy 2017, 2, 780.

[17] Z. Yang, J. Deng, X. Sun, H. Li, H. Peng, Adv. Mater. 2014, 26, 2643.

[18] S. Yoon, D. Khang, ACS Appl. Mater. Interfaces 2018, 11, 3290.

[19] Y. Zhang, S. Xu, H. Fu, J. Lee, J. Su, K. Hwang, J. Rogers, Y. Huang, Soft Matter 2013, 9, 8062.

[20] D. Son, J. Lee, S. Qiao, R. Ghaffari, J. Kim, J. Lee, C. Song, S. Kim, D. Lee, S. Jun, S. Yang, M. Park, J. Shin, K. Do, M. Lee, K. Kang, C. Hwang, N. Lu, T. Hyeon, D. Kim, Nat. Nanotechnol. 2014, 9, 397.

[21] D. Kim, J. Ahn, W. Choi, H. Kim, T. Kim, J. Song, Y. Huang, Z. Liu, C. Lu, J. Rogers, Science 2008, 320, 507.

[22] N. Bowden, S. Brittain, A. Evans, J. Hutchinson, G. Whitesides, Nature 1998, 393, 146.

[23] J. Lee, J. Wu, M. Shi, J. Yoon, S. Park, M. Li, Z. Liu, Y. Huang, J. Rogers, Adv. Mater. 2011, 23, 919.

[24] J. Lee, J. Wu, J. Ryu, Z. Liu, M. Meitl, Y. Zhang, Y. Huang, J. Rogers, Small 2012, 8, 1797

[25] J. Nam, Y. Lee, W. Choi, C. Kim, H. Kim, J. Kim, D. Kim, S. Jo, Adv. Energy Mater. 2016, 6, 21.
[26] R. R. Bahabry, A. T. Kutbee, S. M. Khan, A. C. Sepulveda, I Wicaksono, M. Nour, N. Wehbe, A. S Almislem, M. T. Ghoneim, G. A. Torres Sevilla, A. Syed, S. F. Shaikh, M. M. Hussain, Adv. Energy Mater. 2018, 8, 1702221.

[27] K. Masuko, M. Shigematsu, T. Hashiguchi, D. Fujishima, M. Kai, N. Yoshimura, T. Yamaguchi, Y. Ichihashi, T. Mishima, N. Matsubara, T. Yamanishi, T. Takahama, M. Taguchi, E. Maruyama, S. Okamoto, IEEE J. Photovoltaics 2014, 4, 1433.

[28] M. Lu, S. Bowden, U. Das, R. Birkmire, Appl. Phys. Lett. 2007, 91, 063507.

[29] E. Franklin, K. Fong, K. Mclntosh, A. Fell, A. Blakers, T. Kho, D. Walter, D. Wang, N. Zin, M. Stocks, E. Wang, N. Grant, Y. Wan, Y. Yang, X. Zhang, Z. Feng, P. Verlinden, Prog. Photovoltaics 2016, 24, 411.

[30] K. Yoshikawa, W. Yoshida, T. Irie, H. Kawasaki, K. Konishi, H. Ishibashi, T. Asatani, D. Adachi, M. Kanematsu, H. Uzu, K. Yamamoto, Sol. Energy Mater. Sol. Cells 2017, 173, 37.

[31] T. Rahman, A. To, M. Pollard, N. Grant, J. Colwell, D. Payne, J. Murphy, D. Bagnall, B. Hoex, S. Boden, Prog. Photovoltaics 2018, 26, 38 .

[32] K. Yoshikawa, H. Kawasaki, W. Yoshida, T. Irie, K. Konishi, K. Nakano, T. Uto, D. Adachi, M. Kanematsu, H. Uzu, K. Yamamoto, Nat. Energy 2017, 2, 17032.

[33] T. Xu, Z. Tao, H. Li, X. Tan, H. Li, Adv. Mech. Eng. 2017, 9, 168781401773815.

[34] K. Lee, I. Hwang, N. Kim, D. Choi, H. Um, S. Kim, K. Seo, Nanoscale 2016, 8, 14473.

[35] Y. Zhang, H. Liu, Crystals 2019, 9, 107.

[36] M. Shaeri, R. Bonner, Int. J. Heat Mass Transfer 2019, 131, 1164.

[37] C. Emmott, J. Röhr, M. Campoy-Quiles, T. Kirchartz, A. Urbina, N. Ekins-Daukes, J. Nelson, Energy Environ. Sci. 2015, 8, 1317. 